\title{
The Manifestation of Molecular Double-Well Structure in NO Photoelectron Spectrum
}

\author{
Xu Hua-Wen and Meng Qing-Tian* \\ School of Physics and Electronics, Shandong Normal University, Jinan 250358, China
}

(Received August 17, 2018)

\begin{abstract}
The double-well structure emerges as a result of the potential coupling in a molecular system. In this paper, taking the ionization of $\mathrm{NO}$ as an example, its influence on time-resolved photoelectron spectrum is investigated, by using the time-dependent wave packet method. The results reveal that the double-well structure formed by the crossing of NO excited state potentials supports several degenerate energy levels, which plays a crucial role in determining the dynamical behavior of $\mathrm{NO}$ molecule. It is also demonstrated that by adjusting the delay time of pump and probe laser pulses in the process of NO multiphoton ionization, the influence of double-well structure on time-resolved photoelectron spectrum can be manifested by the variation of vibrational population in the doublewell. The investigation about the effect of double-well structure on time-resolved photoelectron spectrum can provide us with a better understanding of dynamical behavior of molecules in external fields.
\end{abstract}

DOI: 10.12693/APhysPolA.136.118

PACS/topics: double-well, time-resolved photoelectron spectrum, time-dependent wave packet, NO

\section{Introduction}

As a common model in quantum systems, the doublewell structure has received much attention both theoretically and experimentally [1-7]. The influence of relevant parameters, e.g., relative depth and width of the double-well structure, on the wave function and energy levels of the related systems has been well investigated $[8,9]$. Also, some important dynamics, such as the Bose-Einstein condensates and the tunneling process in double-well have been explored [10,11]. All of these above mentioned provide us a good foundation to understand the influence of double-well structure on molecular systems.

As a representative of diatomic molecules, NO molecule is well studied in previous researches, including the photoionization and photodissociation behaviors under intense laser fields [12]. It is found that in the process of NO multiphoton ionization (MPI), some parameters of laser field, such as laser intensity and half-width, etc., play a crucial role [13]. By analyzing the time-resolved photoelectron spectrum (TRPES) of NO molecule, it is shown that the coupling between NO excited potentials is a significant factor of influencing the dynamical behaviors of NO molecule. Especially when the delay time of the pump and probe laser pluses is small, the laser intensity increases, as the overlap of two pluses makes the coupling strength much stronger than that of large delay time, which may lead to some NO molecular "invisible" states becoming visible ones [13]. This kind of coupling can give rise to the avoided crossing of potential curves, and therefore form the double-well structure of the system.

* corresponding author; e-mail: qtmeng@sdnu.edu.cn
As far as we know, there are only few studies showing how the double-well structure affects the dynamical behavior of molecule. In this paper, the time-dependent wave packet (TDWP) method will be used to simulate the process of NO MPI and investigate how the doublewell structure influences the TRPES. The remainder of the paper is arranged as follows. In Sect. 2, the theoretical methods to deal with double-well system are introduced, including the double-well model and the "splitoperator" scheme of wave packet propagation [14-17]. In Sect. 3, the influence of double-well structure on TRPES is discussed and analyzed. Finally, in Sect. 4, the conclusions are presented.

\section{Review of the theoretical treatment}

\subsection{Double-well structure}

In the NO MPI experiment of Ludowise et al. [18], the five-state model is used to analyze the results of the experiment. However, due to the coupling of the potentials, the resulting crossing of the potential curves will inevitably influence the related dynamical behaviors of molecules. Here, for convenient discuss, we choose this kind of crossing (which is also called avoided crossing) as a special form of electronic structure and investigate its manifestation in the TRPES. In calculation, we take the coupling strength $\boldsymbol{E}(\boldsymbol{r})$ in the form of Gaussian function

$$
E(r)=E_{0} \exp \left(r-r_{0}\right) / \sigma^{2},
$$

where $r_{0}$ is the position of cross point, $r$ is the internuclear distance, and the avoided crossing is represented by a double-well model (Fig. 1). In this process of NO MPI with the double-well model, a strong laser pulse pumps a wave packet prepared in the ground electronic state to the double-well; another laser pulse is used to probe the dynamic behavior of the wave packet occurring on the state. 


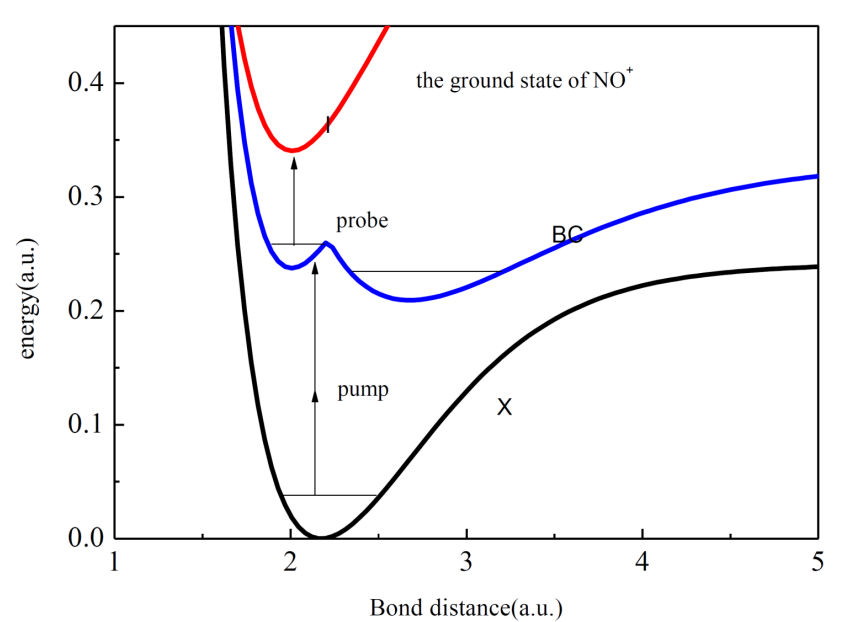

Fig. 1. Double-well model. $\mathrm{X}$ and I denote state $X^{2} \Pi_{\frac{1}{2}}$ of $\mathrm{NO}$ and the ground state of $\mathrm{NO}^{+}$, respectively. $\mathrm{BC}$ denotes the double-well structure of the NO excited state. A first laser pulse pumps a wave packet to an excited electronic state. The wave packet's motion is probed by a second time delay laser pulse, which is also used to ionize the molecule.

The Hamiltonian describing the dynamic behavior of NO molecule in external fields can be written as

$$
\hat{H}=\hat{T}+\hat{V},
$$

where $\hat{T}=-\frac{\hbar}{2 \mu} \frac{\partial^{2}}{\partial r^{2}}$ is the kinetic energy operator and $\hat{V}$ is the potential energy operator. In calculation, we write the potential matrix in double-well model as follows:

$$
\begin{gathered}
\hat{V}(r, t)= \\
\left(\begin{array}{ccccc}
V_{\mathrm{X}} & W_{\mathrm{BCX}} & 0 & \ldots & 0 \\
W_{\mathrm{XBC}} & V_{\mathrm{BC}} & W_{\mathrm{IBC}} & \ldots & W_{\mathrm{IBC}} \\
0 & W_{\mathrm{BCI}} & V_{I}+E_{I, 1} & \ldots & 0 \\
\vdots & \ldots & \ldots & \ldots & \vdots \\
0 & W_{\mathrm{BCI}} & 0 & \ldots & V_{I}+E_{I, n}
\end{array}\right),
\end{gathered}
$$

where $V_{X}$ refers to the potential energy of neutral electronic state $X^{2} \Pi$ and $V_{B C}$ is the double-well potential energy. $E_{I, j}(j=1,2, \ldots, n)$ denote the potential energies of the discretized set of continuum states in the part of ion-pair states, with $V_{I}+E_{I, j}(j=1,2, \ldots, n)$ the electron kinetic energies. $W_{i j}=\mu_{i j} E(t)(i, j=\mathrm{X}$, $\mathrm{BC}, \mathrm{I})$ denote the coupling between the electronic states involved with the dipole matrix elements $\mu_{i j}$ and the external field $E(t)$.

\subsection{Method to solve the time-dependent Schrödinger equation}

The dynamical information associated with the MPI can be obtained by solving the time-dependent Schrödinger equation. Within the Born-Oppenheimer approximation, the time-dependent Schrödinger equation can be written as

$$
\mathrm{i} \hbar \frac{\partial \Psi(r, t)}{\partial t}=\hat{H} \Psi(r, t)
$$

Using the "split-operator" method and denoting the small time interval $\Delta t$ the canonical solution of Eq. (4) can be written as

$$
\Psi(r, t+\Delta t)=\exp \left(\frac{-\mathrm{i}}{\hbar} \hat{H} \Delta t\right) \Psi(r, t)
$$

where $\exp \left(-\frac{\mathrm{i}}{\hbar} H \Delta t\right)$ is the time evolution operator with $\hat{H}=\hat{T}+\hat{V}$. Then, within the second order approximation, the expression of Eq. (5) can be written as

$$
\Psi\left(r, t_{0}+\Delta t\right) \approx\left[U_{T}^{\frac{1}{2}} U_{V} U_{T}^{\frac{1}{2}}\right] \Psi\left(r, t_{0}\right),
$$

where $U_{T}=\exp \left(-\frac{\mathrm{i} \Delta t}{\hbar} \hat{T}\right), U_{V}=\exp \left(-\frac{\mathrm{i} \Delta t}{\hbar} \hat{V}\left(r, t_{0}\right)\right)$ are two new evolution operators, which represent the evolutions of kinetic energy and potential energy, respectively. After propagating $n$ steps, the wave function can be written approximately as

$$
\Psi\left(r, t_{0}+n \Delta t\right) \approx\left[\prod_{k=0}^{n-1} U_{T} U_{V}\left(r, t_{0}+k \Delta t\right)\right] \Psi\left(r, t_{0}\right) .(7)
$$

Once the wave function $\Psi(r, t)$ is determined, the population in each electronic state (including ones in the electron-ion pair states) can be given by

$$
P(t)=\int \mathrm{d} r \Psi^{*}(r, t) \Psi(r, t) .
$$

Evidently, we can describe the population of ionized electrons with $P(t)$ on each state of $\mathrm{NO}^{+}$changing with the kinetic energy of ionized electrons, and then obtain the TRPES.

\section{Results and discussion}

The simulated time-resolved photoelectron spectrum associated with the double-well structure is displayed in Fig. 2. The comparison of these figures shows that when the delay time between the two laser pulses

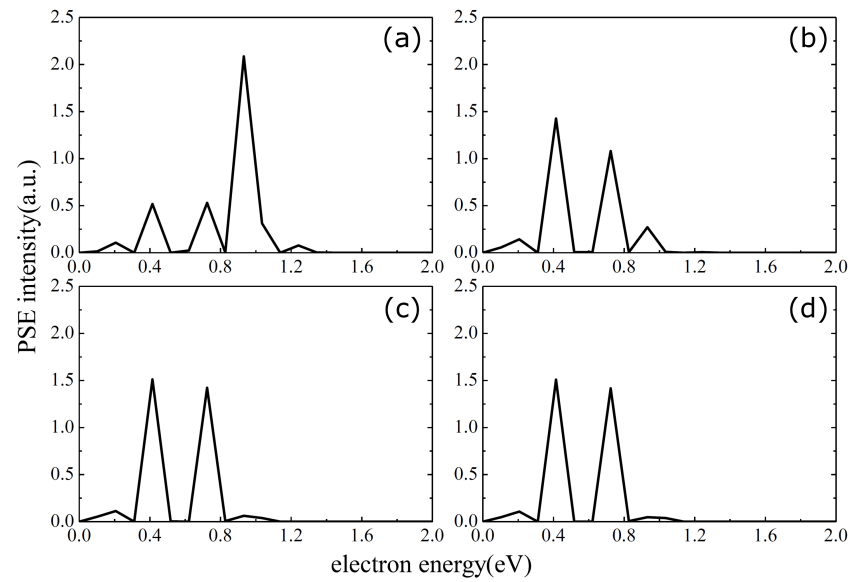

Fig. 2. The femtosecond time-resolved photoelectron spectra from $2+1$ MPI of NO double-well system at fixed pump and probe wavelengths for pump-probe delay times of $0,50,100,200$ fs for (a)-(d). The pump intensity is $8 \times 10^{11} \mathrm{~W} / \mathrm{cm}^{2}$, and the probe intensity is $3 \times 10^{10} \mathrm{~W} / \mathrm{cm}^{2}$. 


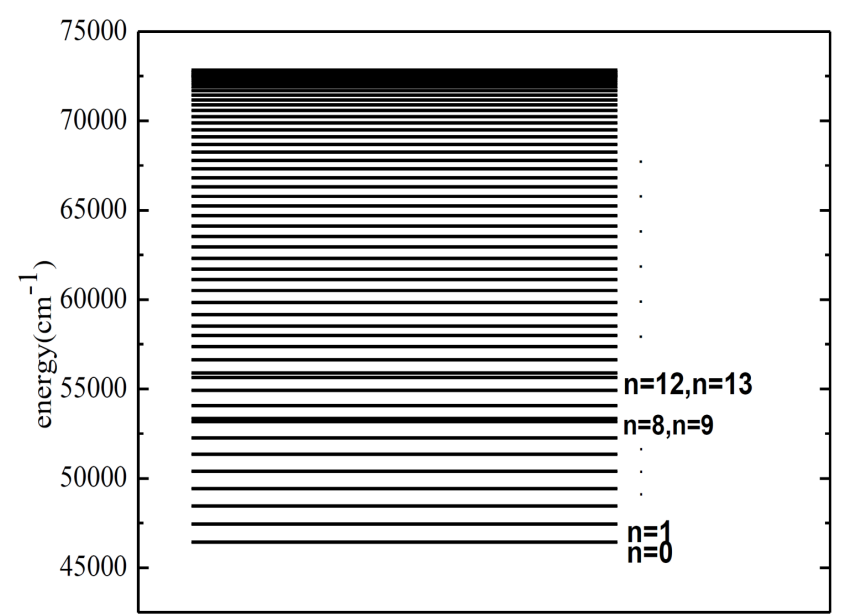

Fig. 3. The energy level in the double-well with $n$ denoting the vibrational quantum number. The 8th and 9th energy levels are degenerate, the 12 th and 13th energy levels are also degenerate.

(360 $\mathrm{nm}$ and $340 \mathrm{~nm}$, respectively) is not zero, there are two high peaks in the picture, while there is one prominent peak when the delay time between the two laser pulses is zero. Apparently, the difference in peak number and height is caused by different delay time. Actually, the reasons behind this are more complicated. Due to the coupling of the potentials, the formed double-well and the corresponding potential barrier give rise to the appearance of several degenerate energy levels, which can lead to the change of the population in the double-well. When the delay time is $0 \mathrm{fs}$, because of the overlap of the pump and probe laser fields, many molecules are ionized, and the kinetic energy of most ionized electrons are near $1.0 \mathrm{eV}$, corresponding to the 13th occupied vibrational energy level in the double-well, which is resonant with the pump laser of $360 \mathrm{~nm}$. When the delay time is $200 \mathrm{fs}$, the absence of the former laser pulse results in the new distribution of molecules in the double-well, and some of the molecules are populated on the 8th and 9th energy levels, which are the lower degenerate energy levels (Fig. 3) in the double-well. This is the reason why the peak appears in position $0.4 \mathrm{eV}$. Furthermore, as is also shown in Fig. 4, the laser intensity is also influential in the process of NO MPI. Obviously, with increase in the laser intensity in small range, the double-peak becomes much higher, which can be regarded as more molecules being ionized from the double-well.

The TRPES of NO double-well system can also be interpreted by the tunneling effect [19]. As can be seen in Fig. 5, the first laser pulse pumps the wave packet to the left part of the double-well. Even if the energy of these molecules is not high enough to penetrate the potential barrier in the double-well, with time evolution, the existence of tunneling effect will lead to some molecules appearing in the right one (Fig. 5). Certainly, molecules in both parts of the double-well, can possibly be ionized. Thus, due to the special structure of double-well,

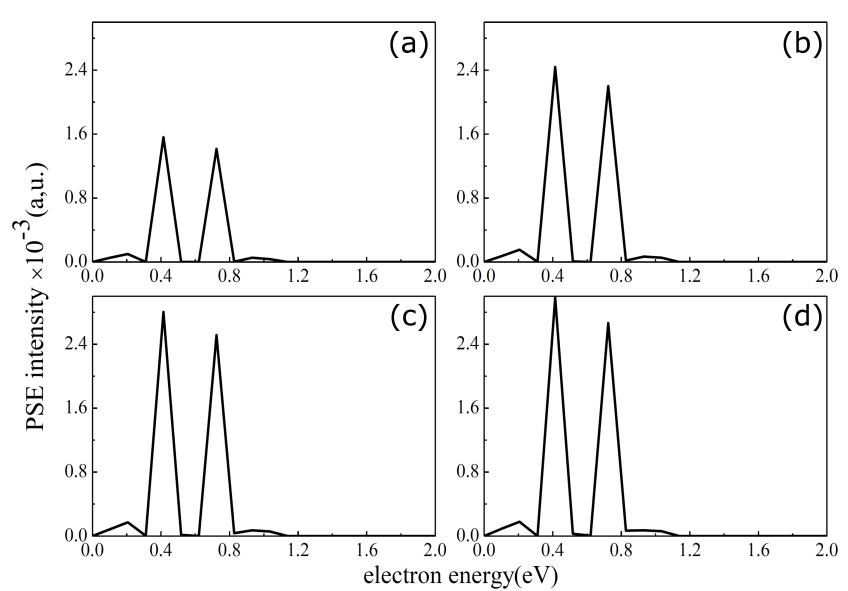

Fig. 4. The comparison of simulated TRPES of NO double-well system with different pump laser intensities. Each of these figures is the result of pump-probe delay time $200 \mathrm{fs}$. The pump intensities are $8 \times 10^{11}, 1.5 \times 10^{12}$, $2 \times 10^{12}, 2.5 \times 10^{12} \mathrm{~W} / \mathrm{cm}^{2}$ for (a)-(d), respectively.

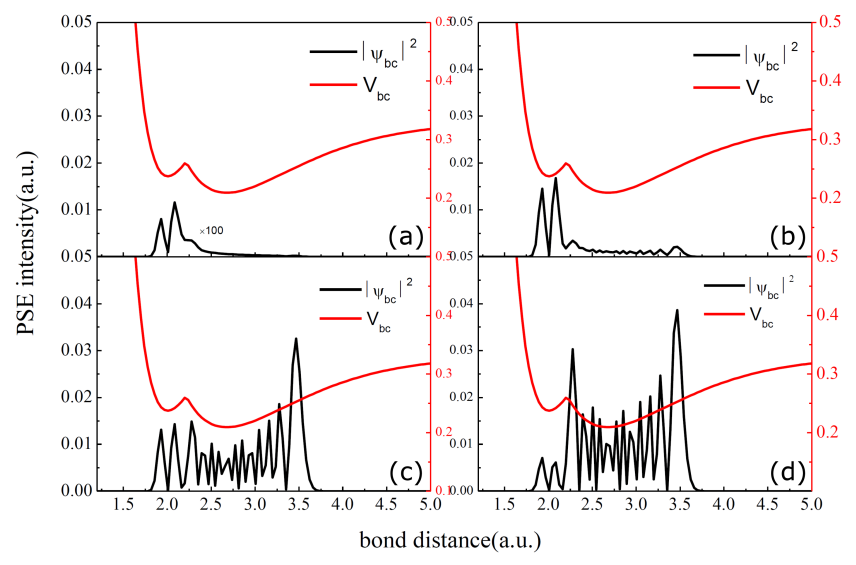

Fig. 5. The comparison of $\Psi_{b c}$ (black line) in $V_{b c}$ (red line) with different evolution times. The evolution time for (a)-(d) is $32,64,96,128 \mathrm{fs}$.

and the corresponding tunneling effect, the population of molecules in the double-well potential is quite different from those in single-well potential. That is why the double-well structure plays an important role in interpreting the ionization dynamics of molecules.

\section{Conclusions}

In this work, using the time-dependent wave packet method, and taking the ionization of NO molecule as an example, we investigate the influence of double-well structure on photoelectron spectrum. It is found that due to the special structure of double-well, the potential supports several degenerate energy levels. Furthermore, when the molecules are excited to the higher degenerate energy levels in the double-well, with time evolution, these molecules are likely to populate in the lower degenerate energy levels, which can be manifested by the double high peaks in the photoelectron spectrum. 
In addition, the potential barrier also greatly influences the motion of the wave packet in the double-well, which can be interpreted by the tunneling effect. Moreover, with increase in the laser intensity in small range, more molecules can be ionized from the double-well. The investigation will be beneficial for the external-field manipulation of atomic and molecular processes.

\section{Acknowledgments}

This work was supported by National Natural Science Foundation of China (No. 11674198).

\section{References}

[1] K.W. Xiao, W.H. Hai, J. Liu, Phys. Rev. A 85, 013410 (2012).

[2] X.F. Zhou, S.L. Zhang, Z.W. Zhou, Phys. Rev. A 85, 023603 (2012).

[3] H. Cartarius, G. Wunner, Phys. Rev. A 86, 013612 (2012).

[4] B.H. Chen, Y. Wu, Q.T. Xie, J. Phys. A Math. Theor. 46, 035301 (2013).

[5] A. Bielas, M.M. Maska, Acta Phys. Pol. A $\mathbf{1 2 6}$ A-118 (2014).

[6] Q. Kui, Z.X. Ni, J. Fuyang Norm. Univ. 32, 38 (2015).
[7] P. Jakubczyk, K. Majchrowski, I. Tralle, Acta Phys. Pol. A 132, 115 (2017).

[8] Z.G. Yang, Z.G. Wang, J. Zhoukou Teachers Coll. 19, $24(2002)$

[9] X.X. Liang, J.G. Li, C.Z. Dong, J. At. Mol. Phys. 25, 1039 (2008).

[10] F. Xu, L. Zhang, J. At. Mol. Phys. 34, 461 (2017).

[11] X.T. Xin, F. Huang, Z.J. Xu, H.B. Li, Chin. Phys. B 7, 284 (2014)

[12] L. Kai, S. Di, Ali Azarm, See-Leang Chin, Fan-ao Kong, Chin. J. Chem. Phys. 23, 252 (2010).

[13] Q.T. Meng, G.H. Yang, H.L. Sun, K.L. Han, N.Q. Lou, Phys. Rev. A 67, 063202 (2003).

[14] A.D. Bandrauk, S. Hai, Chem. Phys. Lett. 176, 428 (1991).

[15] M.D. Feit, J.A. Fleck, J. Chem. Phys. 78, 301 (1983).

[16] J.W. Cooley, J.W. Tuckey, Math. Comput. 19, 297 (1965).

[17] M.D. Feit, J.A. Fleck, A. Steiger, J. Comput. Phys. 47, 412 (1982).

[18] P. Ludowise, M. Blackwell, Y. Chen, Chem. Phys. Lett. 258, 530 (1996).

[19] G. Xu, J. Chongqing Three Gorges Univ. 3, 101 (2006). 